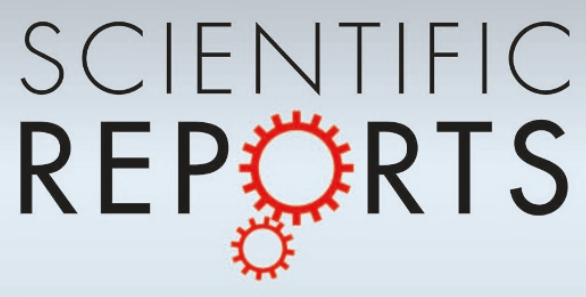

OPEN

SUBJECT AREAS:

APPLIED PHYSICS

ELECTRONICS, PHOTONICS AND DEVICE PHYSICS

Received

12 February 2014

Accepted

4 March 2014

Published

9 April 2014

Correspondence and requests for materials should be addressed to

M.-H.L. (luminghui@ nju.edu.cn)

\title{
Accidental degeneracy of double Dirac cones in a phononic crystal
}

\author{
Ze-Guo Chen' ${ }^{1}$ Xu Ni' ${ }^{1}$, Ying Wu' ${ }^{2}$, Cheng He' , Xiao-Chen Sun', Li-Yang Zheng' ${ }^{1}$ Ming-Hui Lu' \\ \& Yan-Feng Chen'
}

${ }^{1}$ National Laboratory of Solid State Microstructures \& Department of Materials Science and Engineering, Nanjing University, Nanjing 210093, People's Republic of China, ${ }^{2}$ Division of Mathematical and Computer Sciences and Engineering, King Abdullah University of Science and Technology (KAUST), Thuwal 23955-6900, Saudi Arabia.

Artificial honeycomb lattices with Dirac cone dispersion provide a macroscopic platform to study the massless Dirac quasiparticles and their novel geometric phases. In this paper, a quadruple-degenerate state is achieved at the center of the Brillouin zone in a two-dimensional honeycomb lattice phononic crystal, which is a result of accidental degeneracy of two double-degenerate states. In the vicinity of the quadruple-degenerate state, the dispersion relation is linear. Such quadruple degeneracy is analyzed by rigorous representation theory of groups. Using $\vec{k} \cdot \vec{p}$ method, a reduced Hamiltonian is obtained to describe the linear Dirac dispersion relations of this quadruple-degenerate state, which is well consistent with the simulation results. Near such accidental degeneracy, we observe some unique properties in wave propagating, such as defect-insensitive propagating character and the Talbot effect.

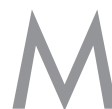

any unique phenomena in graphene such as quantum Hall effect, Zitterbewegung, Klein paradox and pseudo-diffusion, are attributed to the unique dispersion relation of massless quasiparticles solved by Dirac equation ${ }^{1-5}$. The eigen-energy $E$ is linearly proportional to the wave vector $k$ at the six corners of the hexagonal boundary of the Brillouin zone (BZ). The upper and lower bands near the $\mathrm{K}$ point act as two cones touching at one degenerate point, which is the so-called Dirac point and such conical dispersion is called a Dirac cone. Compared to Dirac cone dispersion at the corner of the BZ in graphene or photonics and phononics ${ }^{6-10}$, the recent observation of Dirac cones at the center of the BZ in photonic and phononic crystals (PC) has also attracted much attention. Under certain circumstances, those Dirac cones can be mapped into a zero-refractive-index material, whose parameters (e.g. permittivity and permeability in electromagnetics, effective mass density and reciprocal of bulk modulus in acoustics) are both vanishing ${ }^{11-15}$. It provides a new method to achieve zero-index materials with simple photonic and phononic crystals so that many interesting properties such as wave shaping and cloaking are easily demonstrated ${ }^{12,13,16,17}$.

Linear dispersion is a key feature of a Dirac cone. However, the linear dispersion at a finite frequency is in general forbidden at the center of the BZ, because of time-reversal symmetry ${ }^{18,19}$. The previously mentioned linear dispersion relations at the center of the BZ in classical wave systems are achieved by accidental degeneracy. In 2D photonic and phononic crystals with $C_{4 v}$ symmetry ${ }^{11-13}$, it has been demonstrated that the accidental degeneracy of a monopolar state (or a quadrupolar state) and a double-degenerate dipolar state can lead to three-folded degeneracy showing linear dispersion in the vicinity of the $\Gamma$ point. In addition to the linear bands, there is a flat band intersecting with them at the Dirac point. This is a major difference from the Dirac cones observed in graphene system, in which only two linear bands touch at the Dirac point. From a perturbation theory, it has been demonstrated that the Dirac cone induced by the triple-degenerated states at the center of the BZ is not a truly a Dirac cone because the reduced Hamiltonian cannot be casted into a Dirac equation (corresponding to three states) and the Berry phase equals to zero. Therefore, to be more precise, it is called a Dirac-like cone $e^{20}$. Recently, the double Dirac cone degeneracy at the $\mathrm{BZ}$ center has been predicted in triangular-lattice metamaterials with $C_{6 \mathrm{v}}$ symmetry ${ }^{21}$. However, to the best of our knowledge, such quadruple-degenerate linear dispersion is still not realized in ordinary dielectric photonic crystals or phononic crystals, and furthermore, the underlying physics, such as the reduced Hamiltonian and the Berry phase, still remains unexplored. Meanwhile, such quadrupledegenerate Dirac-like cone states may also be expected to have rich physics that give rise to unique wave propagating properties to be explored.

In this paper, we demonstrate that a quadruple-degenerate state can be created at the BZ center by accidental degeneracy of $E_{1}$ and $E_{2}$ modes in a two-dimensional phononic crystal with honeycomb lattice. In the vicinity of 

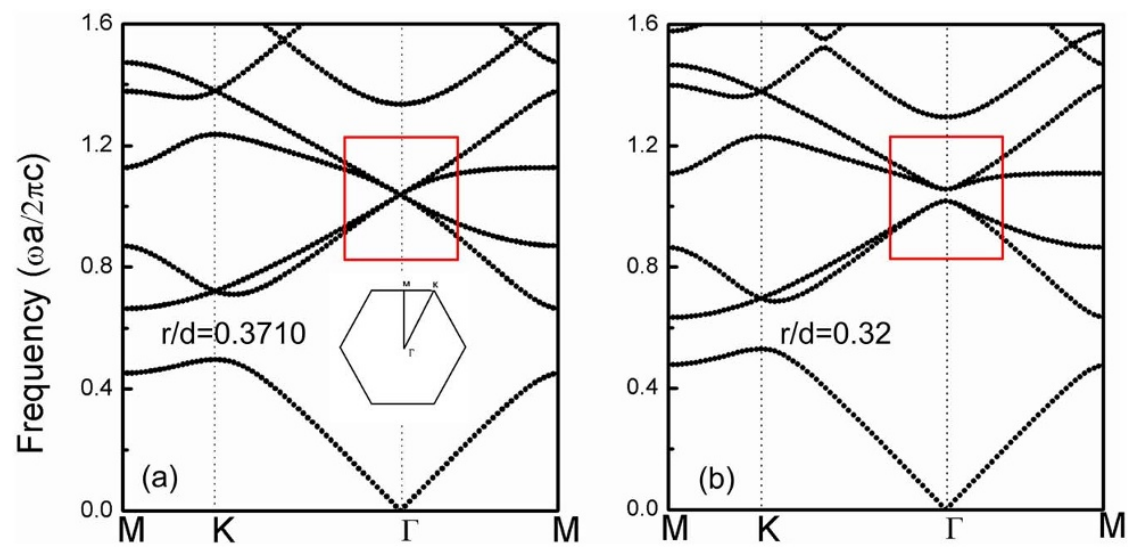

Figure $1 \mid$ (a) Band structures of a 2D honeycomb lattice PC consisting of iron cylinders (radius $r=0.3710 d$ ) in water. Four linear bands intersect at one point of $\omega_{0}=1.0378 \cdot(2 \pi c / a)$ in red rectangle region. (b) Band structure with cylindrical radius $r=0.32 d$, the degeneracy is lift.

the quadruple-degenerate state, the dispersion relation is linear, with four cones touching at their vertices. Different from the Dirac-like cone induced by triple-degenerate state, there is no flat branch. We perform a symmetry analysis to prove the linearity of the dispersion relation and employ a $\vec{k} \cdot \vec{p}$ method to accurately predict the slope of the linear dispersion. The results of the $\vec{k} \cdot \vec{p}$ method also unambiguously reveal that the reduced Hamiltonian can be mapped into a $4 \times 4$ massless Dirac equation but the Berry phase cancels out due to the absence of imaginary part in Dirac equation. Moreover, based on such quadruple Dirac-like degeneracy, a novel defect-insensitive propagating phenomenon and the Talbot effects in such phononic crystals are well described with the acoustic field distribution obtained by the finite element simulation.
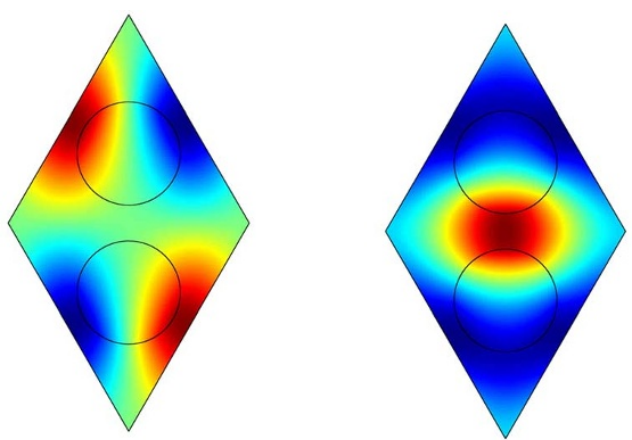

(a)
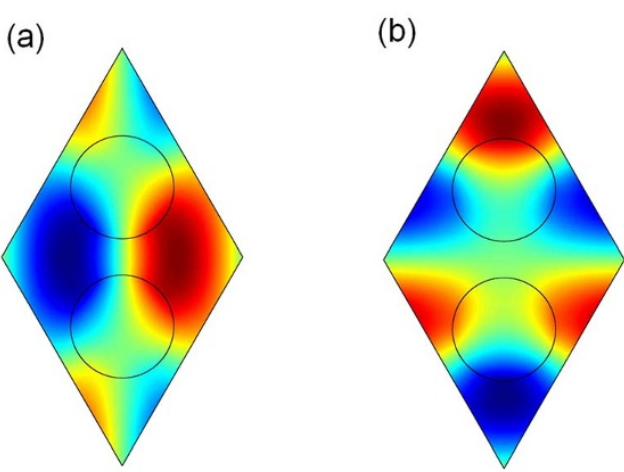

(c)

(d)

Figure $2 \mid$ (a-d) Pressure field distributions of four degenerate Bloch states at $\Gamma$ point as indicated in Fig. 1(a) corresponding to $\psi_{1}, \psi_{2}, \psi_{3}$ and $\psi_{4}$ from low band to high, respectively. Dark red and dark blue colors denote the positive and negative values.

\section{Results}

The 2D PC considered here is composed of a honeycomb array of iron cylinders embedded in water $\left(\rho_{1}=1000 \mathrm{~kg} / \mathrm{m}^{3}, c_{1}=1490 \mathrm{~m} / \mathrm{s}\right.$ and $\rho_{2}=7670 \mathrm{~kg} / \mathrm{m}^{3}, c_{2}=6010 \mathrm{~m} / \mathrm{s}$, where $\rho$ and $c$ denote mass density and velocity of sound and subscripts 1 and 2 correspond to water and iron, respectively). The distance between two cylinders in one unit cell is $d=1 \mathrm{~m}$, the lattice constant is $a=\sqrt{3} m$ and the radius of the cylinder is $r=0.3710 \mathrm{~m}$. Because of the large difference in sound velocities between iron and water, the shear modes inside the iron cylinders can be ignored ${ }^{22,23}$.

Figure 1(a) shows the band structure of the PC. It exhibits four bands touching linearly at one point at the frequency $\omega_{0}=$ 892.77 Hz at the center of the BZ, forming four cones. Such double Dirac cones are resulted from accidental degeneracy, which is clearly demonstrated in Fig. 1(b) when the radii of the cylinders are changed to $r=0.32 \mathrm{~m}$. The quadruple-degenerate state shown in Fig. 1(a) is splitted into two double-degenerate states and the linear dispersion disappears. Since we are interested in the linear dispersion near the $\Gamma$ point, we choose a region denoted by the red rectangle shown in Fig. 1 as our focus. Different from the triply degenerate case ${ }^{11}$, there is no flat branch intersection in our model. Four cones are formed by the linear branches and touches at one point at the frequency of $\omega_{0}=$ $892.77 \mathrm{~Hz}$ with tolerance of $10^{-6}$. These four eigen degenerate states are shown in Figs. 2(a-d).

Firstly, we employ the group theory to analyze the band structure. By examining the symmetry of the eigenstates at the degenerate point, one can check whether the dispersion near that point is linear or not ${ }^{24}$. According to the group theory, the Bloch states at $\Gamma$ point with $C_{6 v}$ symmetry can be described as the basis of the irreducible representation based on the symmetry properties of the states ${ }^{25}$. The four eigen states match well with the four Bloch basis functions as shown in Table 1. The two double-degenerated states which result in the quadruple-degenerate state when they meet together correspond to $E_{1}$ and $E_{2}$ irreducible representations respectively. When any symmetry operation of $C_{6 v}$ is performed, the eigenfunction of $E_{1}$ state transforms like $x$ and $y$, and $E_{2}$ state transforms like $2 x y$ and

Table 1 | Four states at $\Gamma$ point corresponding to four Bloch bases classified under different symmetry operation of $C_{6 v}$ group. $\psi_{1}-\psi_{4}$ correspond to field distributions in Figs. 2(a-d), respectively

\begin{tabular}{llrr}
$C_{6 v}$ & \multicolumn{1}{c}{ Basis } & $\sigma_{x}$ & $\sigma_{y}$ \\
\hline$E_{1}$ & $\psi_{3}, x$ & -1 & 1 \\
$E_{2}$ & $\psi_{4}, y$ & 1 & -1 \\
& $\psi_{1}, x y$ & -1 & -1 \\
& $\psi_{2,}, x^{2}-y^{2}$ & 1 & 1 \\
\hline
\end{tabular}


$x^{2}-y^{2}$. The wave functions near the degenerated point can be expressed as linear combinations of the degenerate states. (These linear combination wave functions are also adopted in $\vec{k} \cdot \vec{p}$ method in the next section.) It should be noted that even under the same operation, the transformation of the Bloch state constructed of $E_{1}$ is different from that of $E_{2}$. Under the symmetry operations of $\sigma_{\mathrm{x}}$ and $\sigma_{y}, E_{1}$ and $E_{2}$ representation can further be classified into four states. Consequently, the double Dirac cone induced by the accidental degeneracy shown in Fig. 1(a) is supported by the group theory analysis $^{21}$. The states near the Dirac point are linear combinations of the Bloch states with parity of $\sigma_{x}$ or $\sigma_{y}$, and the wave equation should possess the same parity. Moreover, considering the compatibility relation along $\Gamma \mathrm{K}$ and $\Gamma M$ directons, $E_{1}=A+B, E_{2}=A+B$, where $A$ is full symmetry representation which indicates the existence of isotropic linear dispersion ${ }^{25}$.

Then, we resort to the well-known $\vec{k} \cdot \vec{p}$ method in electronics to analyze our phononic model ${ }^{20}$. We can rewrite the Bloch functions near $\vec{k}_{0}$ as linear combinations of four $\vec{k}_{0}$ states. Substituting such function into wave equation with periodic boundary conditions, we can get $^{20}$

$$
\operatorname{det}\left|H-\frac{\omega_{n \vec{k}}^{2}-\omega_{j 0}^{2}}{c_{1}^{2}} I\right|=0
$$

where $n$ denotes the band index, $\vec{k}$ is the Bloch wave vector, and $H$ is the reduced Hamiltonian matrix with element $H_{i j}=i \vec{k} \cdot \vec{L}_{i j}, i$ and $j$ are subscripts of matrix elements. Here, $\vec{L}_{i j}$ is a real vector in $x-y$ plane. The $x$ component of $\vec{L}_{i j}$ can be numerically calculated from the Bloch states as,

$L_{i j}(x)=\frac{(2 \pi)^{2}}{\Omega}\left(\int_{\text {unitcell }} \psi_{\vec{k}_{0}}^{*}(\vec{r}) \times \frac{2 \frac{\partial \psi_{\vec{j} 0_{0}}^{*}(\vec{r})}{\partial x}}{\rho_{r}(\vec{r})} d \vec{r}+\oint \psi_{\vec{i}_{0}}^{*}\left(\vec{r}_{0}\right) \times \frac{\rho_{r}-1}{\rho_{r}} \times \cos (\theta) \times \psi_{\vec{j}_{0}}\left(\vec{r}_{0}\right) d \vec{r}_{0}\right),(2)$

where $\rho_{r}(\vec{r})=\rho(\vec{r}) / \rho_{1}, \theta$ is the integration variable. The $y$ component of $\vec{L}_{i j}$ can be calculated using the same process. In Eq. (2), only eight vectors are nonzero. Considering the anti-symmetrical property: $\vec{L}_{i j}=-\vec{L}_{j i}$, only four vectors are independent, and the relationships of these four vectors can be described as [shown in Fig. 3(a)]

$$
\begin{aligned}
& \vec{L}_{13}=-\vec{L}_{24}, \vec{L}_{23}=\vec{L}_{14}, \vec{L}_{13} \cdot \vec{L}_{23}=0 \\
& \vec{L}_{13}=(-0.03768,4.0005), \vec{L}_{14}=(4.0009,0.03768) \\
& \vec{L}_{23}=(4.0011,0.03766), \vec{L}_{24}=(0.03766,-4.0014) .
\end{aligned}
$$

Thus, the reduced Hamiltonian $H$ can be casted into:

$$
H=\left|\begin{array}{cccc}
0 & 0 & i \vec{k} \cdot \vec{L}_{13} & i \vec{k} \cdot \vec{L}_{14} \\
0 & 0 & i \vec{k} \cdot \vec{L}_{23} & i \vec{k} \cdot \vec{L}_{24} \\
-i \vec{k} \cdot \vec{L}_{13} & -i \vec{k} \cdot \vec{L}_{23} & 0 & 0 \\
-i \vec{k} \cdot \vec{L}_{14} & -i \vec{k} \cdot \vec{L}_{24} & 0 & 0
\end{array}\right| .
$$

For the Bloch state at $\vec{k}$ in the vicinity of the $\Gamma$ point, the angle between the Bloch wave vector and $\vec{L}_{13}$ is $\theta$, by using Eq. (1), we can get dispersion relations of two Dirac cones

$$
\frac{\Delta \omega}{\Delta k}= \pm \frac{|\vec{L}| c_{1}^{2}}{2 \omega_{0}}
$$

$\Delta \omega=\omega-\omega_{0}$ and $\Delta k=k-k_{0}$. Eq. (5) is linear in $\Delta k$ and is independent of $\theta$ indicating the isotropy of the dispersion relation, which could be confirmed by the numerical simulations shown by solid dots in Fig. 3(b), and the isotropic equi-frequency contours (EFCs) shown in Figs. 4(b1) and (b2) result from the coupling of the degenerate Bloch state ${ }^{21}$, which match well with the prediction of the group theory.

Knowing the length of $\vec{L}_{13}$, we can analytically calculate the dispersion relations from Eq. (5) as the red lines shown in Fig. 3(b), which overlaps with the solid dots well in the BZ center. It should be noted that although Eq. (5) exhibits only two roots, there should be four solutions to $\mathrm{Eq}$ (1), which means each root represented in Eq. (5) corresponds to two identical (degenerate) solutions. This is an important result, as it indicates that rather than having Dirac cones with different linear slopes, the Dirac cones produced here by the quadruple-degenerate state have identical slopes. This theoretical prediction is consistent with the simulated band structure [shown in Fig. 3(b)]. The equivalent frequency contours (EFCs) of these four bands are plotted in Fig. 4. Near the Dirac point, there is only one circle in the EFCs, verifying the isotropic property [Figs. 4(b1) and 4(b2) are identical]. Away from the Dirac point, apparently seen from Figs. 4(a) and 4(c), the EFCs for different bands are different and their hexagonal shapes indicate the anisotropy of the dispersion.

The linear dispersion at the $\Gamma$ point described above is very similar to the Dirac point at the BZ corner studied earlier ${ }^{5}$. It has been reported that in a phononic crystal the Dirac point at the corner of the BZ carries nonzero Berry phase ${ }^{26}$, while the Dirac-like point with triple degeneracy at $\Gamma$ point carries zero Berry phase ${ }^{20}$. Now, we have achieved double Dirac cone at the $\Gamma$ point by quadruple-degenerate state, does it carry zero or nonzero Berry phase? To answer this question, we perform the following analysis.
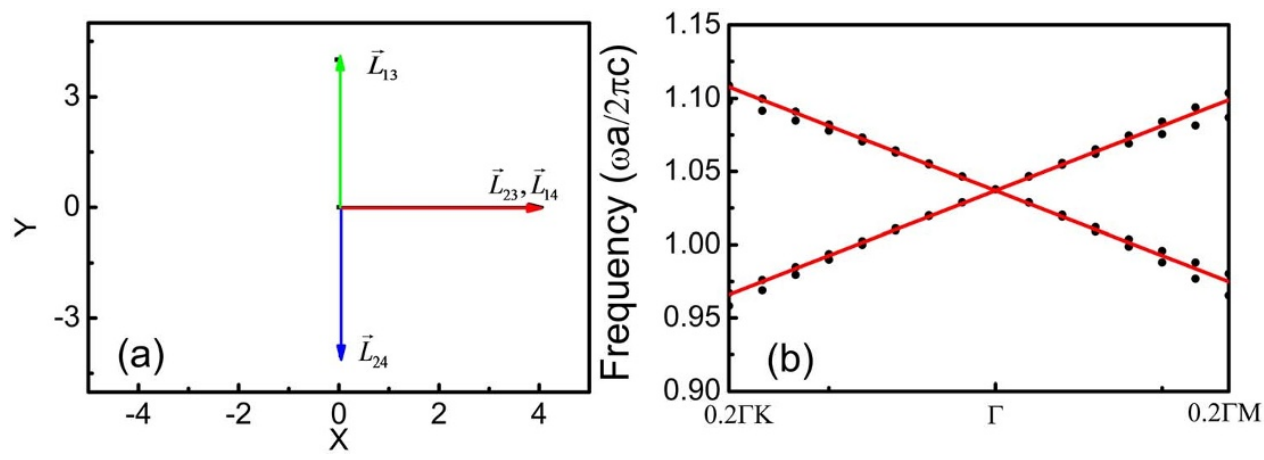

Figure $3 \mid$ (a) The relations of four vectors [seen in Eq.3] in real space calculated by field distribution in Fig. 2 . These four real vectors have the same length. (b) Dirac dispersion relation. Dots and solid lines represent the simulation results and $\vec{k} \cdot \vec{p}$ method results, respectively. 

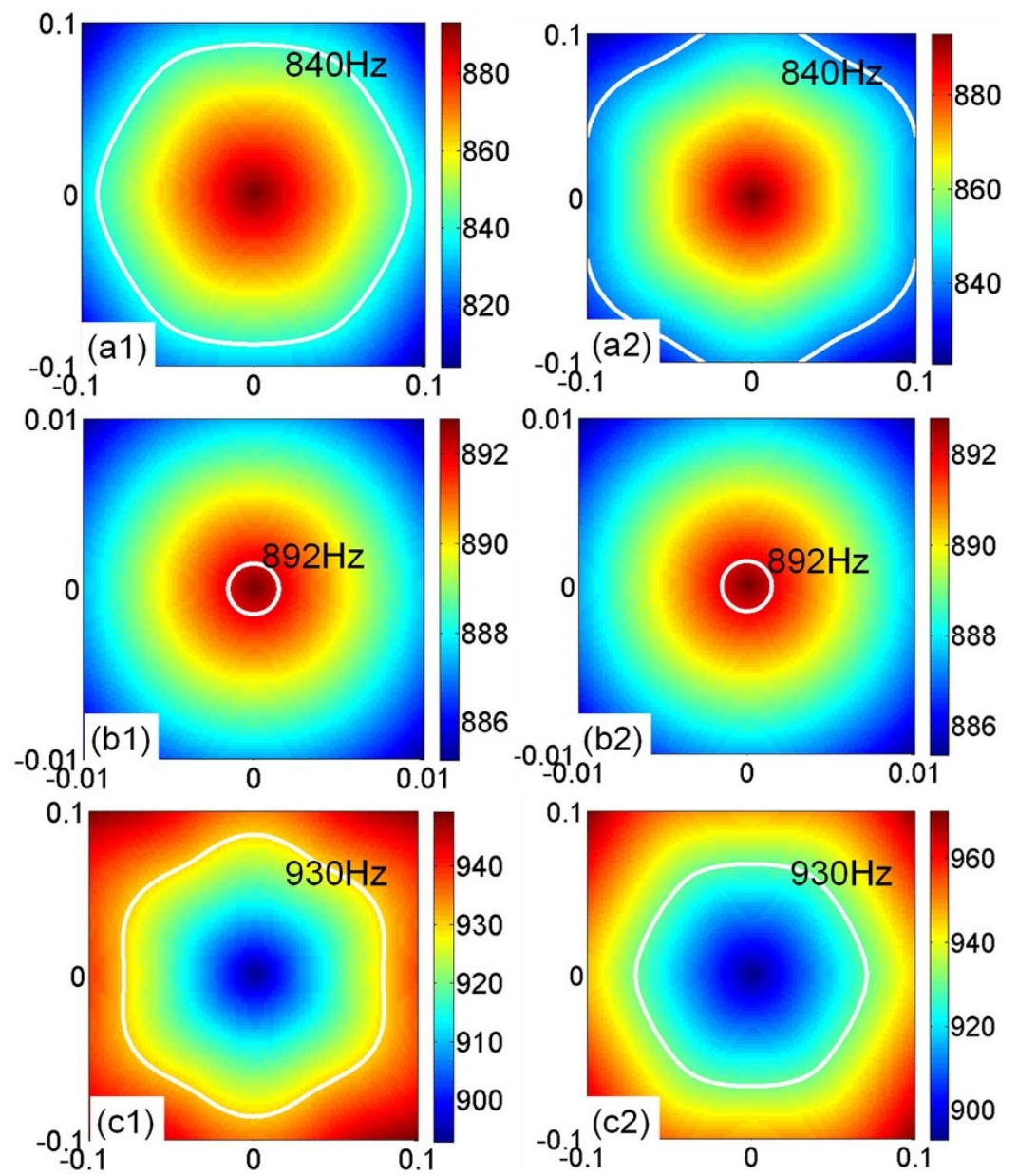

Figure 4 Two EFCs corresponding to different bands at the frequencies of $840 \mathrm{~Hz}, 892 \mathrm{~Hz}, 930 \mathrm{~Hz}$. (a1) and (a2) are EFCs of $840 \mathrm{~Hz}$. (b1) and (b2) are EFCs of $892 \mathrm{~Hz}$ near Dirac points. (c1) and (c2) are EFCs of $930 \mathrm{~Hz}$.

The eigenfunction of the $H$ near $\vec{k}_{0}$ is

$\varphi_{1}(\vec{k})=\frac{1}{\sqrt{2}}\left(\begin{array}{c}i \sin \theta \\ -i \cos \theta \\ 0 \\ 1\end{array}\right) \mathrm{e}^{\mathrm{i} \overrightarrow{\mathrm{k}} \cdot \overrightarrow{\mathrm{r}}} \varphi_{2}(\vec{k})=\frac{1}{\sqrt{2}}\left(\begin{array}{c}i \cos \theta \\ i \sin \theta \\ 1 \\ 0\end{array}\right) \mathrm{e}^{\mathrm{i} \overrightarrow{\mathrm{k}} \cdot \overrightarrow{\mathrm{r}}}$
$\varphi_{3}(\vec{k})=\frac{1}{\sqrt{2}}\left(\begin{array}{c}-i \sin \theta \\ i \cos \theta \\ 0 \\ 1\end{array}\right) \mathrm{e}^{\mathrm{i} \overrightarrow{\mathrm{k}} \cdot \overrightarrow{\mathrm{r}}} \varphi_{4}(\vec{k})=\frac{1}{\sqrt{2}}\left(\begin{array}{c}-i \cos \theta \\ -i \sin \theta \\ 1 \\ 0\end{array}\right) \mathrm{e}^{\mathrm{i} \overrightarrow{\mathrm{k}} \cdot \overrightarrow{\mathrm{r}}}$,

We can calculate the Berry phase as,

$$
\Gamma_{i}=i \cdot \oint\left\langle\varphi_{i}(\vec{k})\left|\nabla_{\vec{k}}\right| \varphi_{i}(\vec{k})\right\rangle \cdot d \vec{k}
$$

Taking $\varphi_{1}(\vec{k})$ as an example, we can write $\sin \theta=\frac{u k_{x}-v k_{y}}{k}$, $\cos \theta=\frac{v k_{x}+u k_{y}}{k}$, where $u^{2}+v^{2}=1$. Substituting $\varphi_{1}(\vec{k})$ into Eq. (7), we find

$$
\begin{aligned}
& \Gamma_{i}=i \cdot \oint\left\langle\varphi_{i}(\vec{k})\left|\nabla_{\vec{k}}\right| \varphi_{i}(\vec{k})\right\rangle \cdot d \vec{k} \\
& =\frac{i}{2} \oint\left[-\frac{i}{k}\left(u k_{x}-v k_{y}\right), \frac{i}{k}\left(v k_{x}+u k_{y}\right), 0,1\right] \\
& \times\left\{\left[\begin{array}{c}
\frac{i}{k}(u \vec{x}-v \vec{y}) \\
\frac{-i}{k}(v \vec{x}+u \vec{y}) \\
0 \\
0
\end{array}\right]+\left[\begin{array}{c}
\frac{i}{k}\left(u k_{x}-v k_{y}\right) \\
\frac{-i}{k}\left(v k_{x}+u k_{y}\right) \\
0 \\
1
\end{array}\right] i \vec{r}\right\} \cdot d \vec{k} \\
& =\frac{i}{2} \oint\left[\frac{1}{k^{2}}\left(k_{x} d k_{x}+k_{y} d k_{y}\right)\right]+\frac{i}{2} \oint 2 i \vec{r} d \vec{k} \\
& =0 \text {, }
\end{aligned}
$$

The same result can be obtained if we use any $\varphi_{i}(\vec{k})$ in Eq. (7), which means the Berry phase for our system at $\Gamma$ point is zero.

In other words, the $H$ of our system can be written as

$$
H=-\vec{L}_{14} \cdot \vec{k} \sigma_{y} \otimes \tau_{x}-\vec{L}_{13} \cdot \vec{k} \sigma_{y} \otimes \tau_{z} .
$$

$\sigma_{\mathrm{y}}, \tau_{\mathrm{x}}, \tau_{\mathrm{z}}$ are all Pauli matrices and two Kronecker product matrices satisfy the anti-commutation relations. Although Eq. (9) is in the form of a massless Dirac equation, $\vec{L}_{14} \cdot \vec{k}$ and $\vec{L}_{13} \cdot \vec{k}$ contain no imaginary parts indicating the zero Berry phase, which is different from the Dirac cone at the corner of the BZ. 

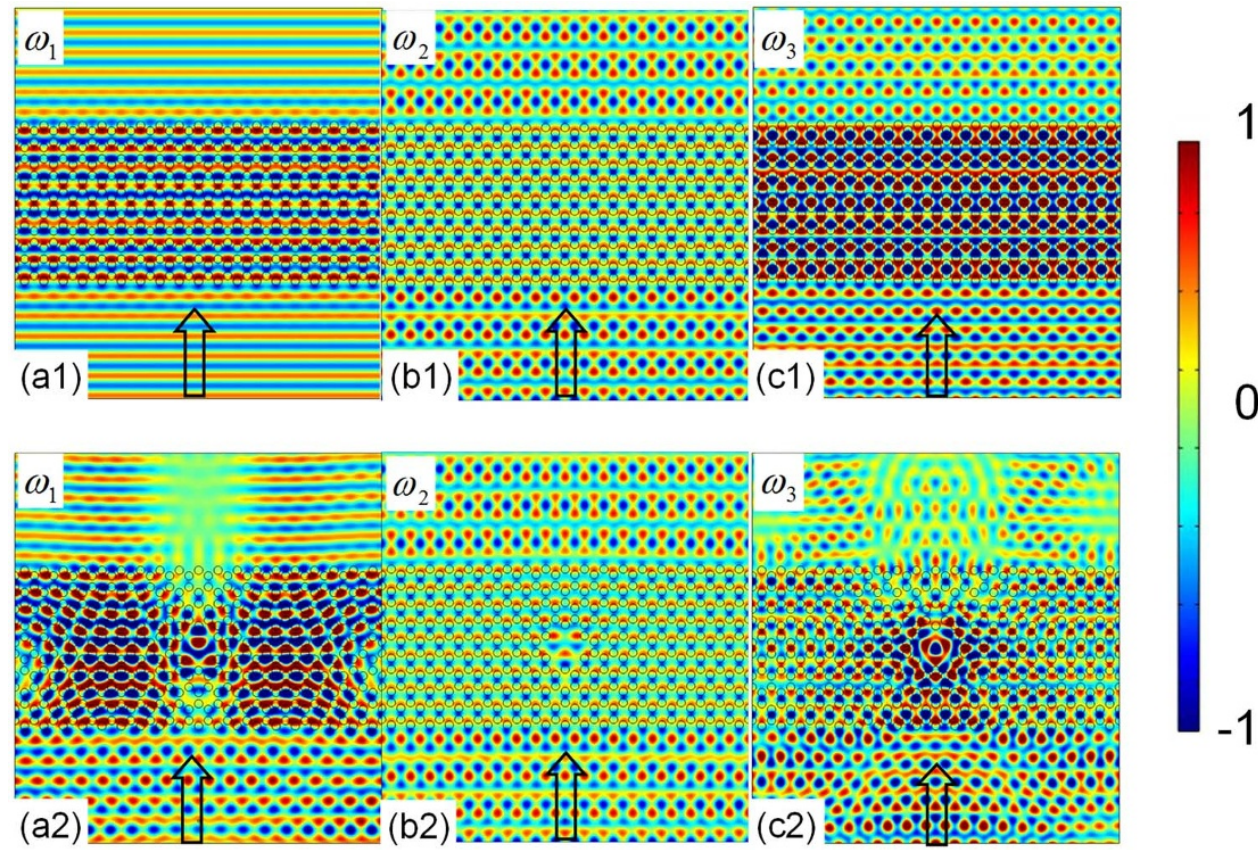

Figure 5 Transmission patterns with plane incidence source. Operation frequencies are set at (a) $\omega_{1}$, (b) $\omega_{2}$ (c) $\omega_{3}$. The suffix 1 or 2 represents the case of PC without or with defect, respectively. (b1) and (c1) exhibit the Talbot effect. (b2) shows the defect-immune property.

Finally, we analyze the wave propagating properties in our system near our concerned frequency. Figure 5 shows the numerical simulations of the wave propagating properties in the PC. In panels al and a2, we set the operating frequency to be $\omega_{1}=0.9409 \omega_{0}$ below the frequency of Dirac point, $\omega_{2}=0.9991 \omega_{0}$ is used in panels b1 and b2 near $\omega_{0}, \omega_{3}=1.0417 \omega_{0}$ is used in panels $c 1$ and $c 2$. The incident wave is along $\Gamma M$ direction. Figure $5(\mathrm{a} 1)$ shows that the outgoing wave preserves the plane wave front, while Fig. 5(b1) shows the Talbot effect ${ }^{27}$. The Talbot effect is a near-field diffraction effect which was first observed in the year of $1836^{28}$, and in this effect a plane wave transmits through a grating or other periodic structures with the resulting wave fronts propagating in such a way that replicates the structure. According to the field distribution shown in Fig. 5(b1), 5(c1), the wave fronts out of the PC share almost the same shape, while only Fig. 5(a1) returns into a plane wave after propagating about 2.3 wavelengths distance. Noted that the widely used effective medium theory is no longer applicable at such high frequency, and we cannot expect a plane wave at frequency $\omega_{2}$ in the PC with $C_{6 v}$ symmetry. Here, $\omega_{1}$ is a threshold frequency to reconstruct the plane wave. For a slight blue shift of frequency $\omega_{1}$, we can find Talbot effect in our system as shown in Fig. 5(c1).

The defect insensitivity of the Talbot effect in our PC is also investigated and the results are shown in Figs. 5(a2), 5(b2) and 5(c2). Comparing with Dirac point at $K$ point or Dirac-like point at $\Gamma$ point
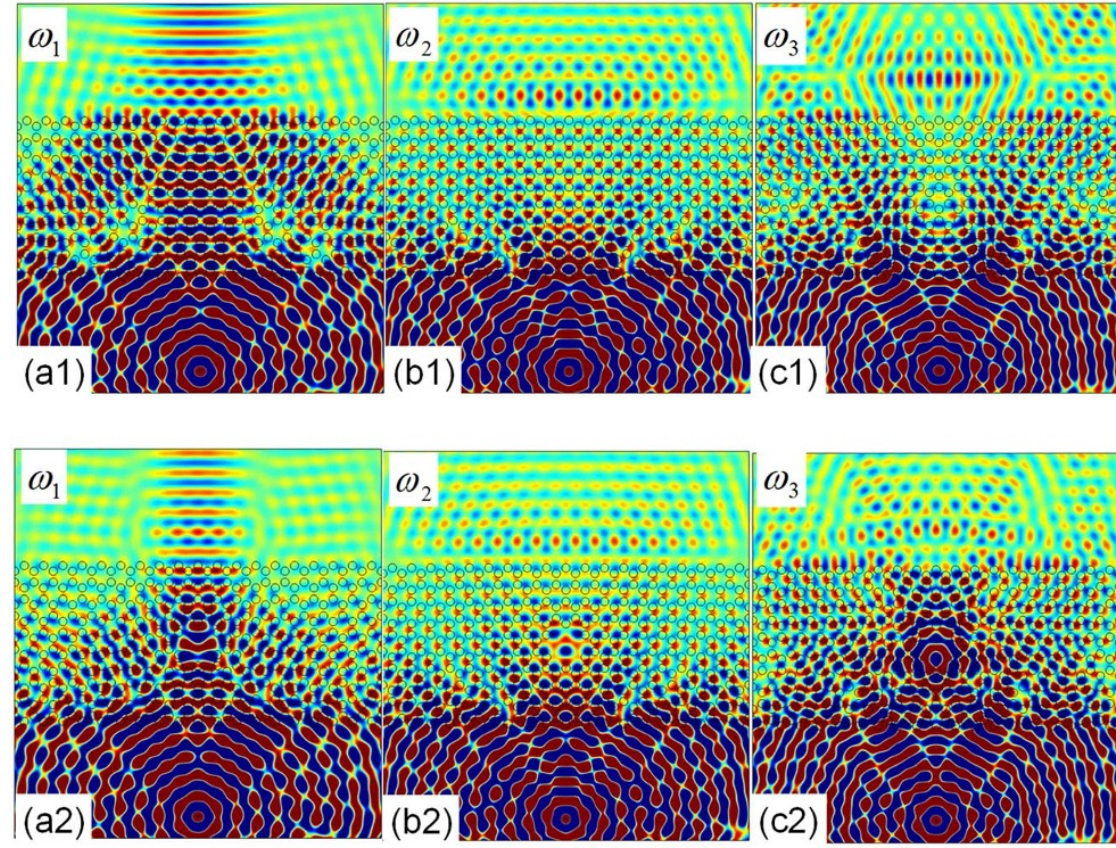

Figure 6 Transmission patterns with cylindrical incidence source. Operation frequencies are set at (a) $\omega_{1}$, (b) $\omega_{2}$, (c) $\omega_{3}$. The suffix 1 or 2 represents the case of PC without or with defect. (b1) and (c1) exhibit the Talbot effect. (b2) shows the defect-immune property. 


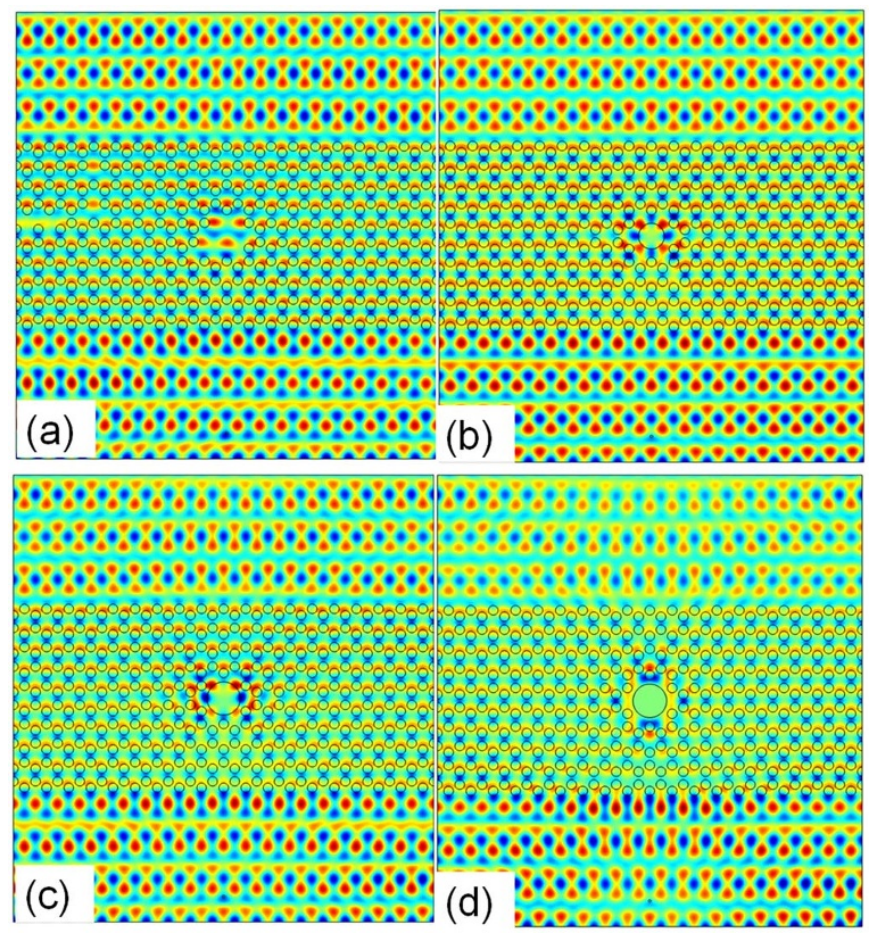

Figure $7 \mid$ Plane wave transmission pattern with various kinds of defects. (a) Random distributed defects. A metallic cylindrical defect of (b) $r=1 \mathrm{~m}$ and (c) $r=1.3 \mathrm{~m}$. (d) An air bubble defect $(r=1.3 \mathrm{~m})$. All of them show a Talbot effects regardless of the type of defects.

in triangular lattice, the propagation of wave in our PC at Dirac cone frequency is more insensitive to defects. At the frequency of $\omega_{2}$, the defect cannot be detected from the transmitted pattern, while it can be easily found at the frequencies of $\omega_{1}$ and $\omega_{3}$. According to the field distributions shown in Fig. 5(a2) and 5(c2), more than one mode are excited in the PC at the frequencies of $\omega_{1}$ and $\omega_{3}$. These modes are all attributed by the scattering of the defect, which would provide various scattering wave vectors. As the EFC shown in Figs. 3(b1) and 3 (b2), it is a circle near the frequency $\omega_{0}$ compared to two big hexagons at the frequencies of $\omega_{1}$ and $\omega_{3}$. Considering the incident angle dependence, one wave vector of incident waves would often excite two outgoing modes near $\omega_{0}$, however, such two outgoing modes would share the same Bloch wave vector due to the double degeneracies [shown in Fig. 1]. Furthermore, the existence of defect contributes to wave vectors with various directions. Thus, we can conclude that these two states near $\omega_{0}$ are insensitive to incidence direction of wave vectors ${ }^{29}$. But, at the frequencies of $\omega_{1}$ and $\omega_{3}$, two non-degenerated modes are excited corresponding to two different Bloch wave vectors, which are dependent on the incidence angle.

To check the angle dependent propagation properties in our system, we employ a cylindrical incidence source which can provide various wave vectors [shown in Fig. 6]. Similar to the case with the plane-wave incidence source, Fig. 6(b1) also shows a Talbot effect. The defects in this system cannot be detected at the frequencies near $\omega_{0}$ [shown in Fig. 6(b2)], which can be regarded as a type of cloaking $^{11,12}$. The negative refraction also can be realized in Fig. $6(\mathrm{a} 1)^{30,31}$.

Moreover, the Talbot effect is immune to various types of defects instead of special cases. Figure 7(a) shows the case of a random distribution with several defects, and the transmission pattern is similar to Fig. 5(b1). With metallic defects $\left(\rho_{2}=7670 \mathrm{~kg} / \mathrm{m}^{3}, c_{2}=\right.$ $6010 \mathrm{~m} / \mathrm{s}, r=1 \mathrm{~m}$ in Fig. 7(b) and $r=1.3 \mathrm{~m}$ in Fig. 7(c)), the scattering of the cylinder is well suppressed. To further demonstrate the ability to reduce the scattering cross section, we introduce an air bubble $(r=1.3 \mathrm{~m})$ which has a strong scattering field in usual underwater acoustic system shown in Fig. 7(d). The scattering of the air bubble is also effectively suppressed.

\section{Discussion}

In summary, we have designed a two-dimensional phononic honeycomb lattice to achieve a quadruple-degenerate state at $\Gamma$ point which is constructed by the accidental degeneracy of two doubledegenerate states. In the vicinity of the quadruple-degenerate state, there exist double isotropic linear Dirac cones. The linear dispersion induced by the accidental degeneracy is rigorously analyzed by the group representation theory. By using the $\vec{k} \cdot \vec{p}$ method, a $4 \times 4$ reduced Hamiltonian is obtained to describe the massless Dirac linear dispersion relation. The Berry phase of such double Dirac cones cancels out due to the absence of the imaginary part. Although there is no flat band in our system, and it neither satisfies long wave approximation nor is regarded as effective zero-index medium, a new kind of novel Talbot effect can still be found in this phononic crystal near the quadruple degenerate point due to the linear and isotropic dispersion, which is insensitive to various types of defects and wave source. The Zitterbewegung is also expected for such quadruple-degenerate state associated with the Dirac equation ${ }^{32,33}$. The enhancement of the nonlinearity is also prospective for the phase matching effect in our system ${ }^{34,35}$.

\section{Methods}

Throughout the paper, the Finite Element Method (FEM) based on commercial software COMSOL Multiphysics is employed for the numerical computations and the simulations. The materials applied in simulations are water and steel. Plane wave radiation boundary conditions are set on the outer boundaries of simulation domain so there will be no interference from the reflected acoustic wave and the periodic boundary condition are employed in the left and right boundaries to simulate the PC with infinite size. The largest mesh element size is set lower than $1 / 20$ of the shortest wavelength.

1. Zhang, Y. B., Tan, Y. W., Stormer, H. L. \& Kim, P. Experimental observation of the quantum Hall effect and Berry's phase in graphene. Nature 438, 201-204 (2005).

2. Katsnelson, M. I. Zitterbewegung, chirality, and minimal conductivity in graphene. Eur. Phys. J. B 51, 157-160 (2006).

3. Katsnelson, M. I., Novoselov, K. S. \& Geim, A. K. Chiral tunnelling and the Klein paradox in graphene. Nature Phys. 2, 620-625 (2006).

4. Sepkhanov, R. A., Bazaliy, Y. B. \& Beenakker, C. W. J. Extremal transmission at the Dirac point of a photonic band structure. Phys. Rev. A 75, 063813 (2007).

5. Castro Neto, A. H., Guinea, F., Peres, N. M. R., Novoselov, K. S. \& Geim, A. K. The electronic properties of graphene. Rev. Mod. Phys. 81, 109-162 (2009).

6. Torrent, D. \& Sánchez-Dehesa, J. Acoustic analogue of graphene: observation of Dirac cones in acoustic surface waves. Phys. Rev. Lett. 108, 174301 (2012).

7. Plihal, M. \& Maradudin, A. A. Photonic band structure of two-dimensional systems: The triangular lattice. Phys. Rev. B 44, 8565-8571 (1991).

8. Ochiai, T. \& Onoda, M. Photonic analog of graphene model and its extension: Dirac cone, symmetry, and edge states. Phys. Rev. B 80, 155103 (2009).

9. Han, D., Lai, Y., Zi, J., Zhang, Z.-Q. \& Chan, C. T. Dirac Spectra and Edge States in Honeycomb Plasmonic Lattices. Phys. Rev. Lett. 102, 123904 (2009).

10. Bittner, S. et al. Observation of a Dirac point in microwave experiments with a photonic crystal modeling graphene. Phys. Rev. B 82, 014301 (2010).

11. Huang, X., Lai, Y., Hang, Z. H., Zheng, H. \& Chan, C. T. Dirac cones induced by accidental degeneracy in photonic crystals and zero-refractive-index materials. Nature Mater. 10, 582-586 (2011).

12. Liu, F., Huang, X. \& Chan, C. T. Dirac cones at $\mathrm{k}=0$ in acoustic crystals and zero refractive index acoustic materials. Appl. Phys. Lett. 100, 071911 (2012).

13. Liu, F., Lai, Y., Huang, X. \& Chan, C. T. Dirac cones at $\mathrm{k}=0$ in phononic crystals. Phys. Rev. B 84224113 (2011)

14. Moitra, P., Yang, Y., Anderson, Z., Kravchenko, I. I. \& Valentine4, D. P. B. J. Realization of an all-dielectric zero-index optical metamaterial. Nature Photon. 7, 791-795 (2013).

15. Wu, Y., Li, J., Zhang, Z.-Q. \& Chan, C. T. Effective medium theory for magnetodielectric composites: Beyond the long-wavelength limit. Phys. Rev. B 74 085111 (2006).

16. Zhu, X., Liang, B., Kan, W., Zou, X. \& Cheng, J. Acoustic cloaking by a superlens with single-negative materials. Phys. Rev. Lett. 106, 014301 (2011).

17. Zhang, S., Xia, C. \& Fang, N. Broadband acoustic cloak for ultrasound waves. Phys. Rev. Lett. 106, 024301 (2011).

18. Sakoda, K. \& Zhou, H. Analytical study of two-dimensional degenerate metamaterial antennas. Opt. Express 19, 13899-13921 (2011).

19. John, D., Joannopoulos, S. G. J., Joshua N. Winn. \& Robert, D. Meade. Photonic Crystals: Molding the Flow of Light (Princeton University Press, New Jersey, 2008). 
20. Mei, J., Wu, Y., Chan, C. T. \& Zhang, Z. Q. First-principles study of Dirac and Dirac-like cones in phononic and photonic crystals. Phys. Rev. B 86, 035141 (2012).

21. Sakoda, K. Double Dirac cones in triangular-lattice metamaterials. Opt. Express 20, 9925-9939 (2012).

22. Kafesaki, M. \& Economou, E. N. Multiple-scattering theory for three-dimensional periodic acoustic composites. Phys. Rev. B 60, 11993-12001 (1999).

23. Li, J. \& Chan, C. T. Double-negative acoustic metamaterial. Phys. Rev. E 70, 055602 (2004)

24. Li, Y., Wu, Y., Chen, X. \& Mei, J. Selection rule for Dirac-like points in twodimensional dielectric photonic crystals. Opt. Express 21, 7699-7711 (2013).

25. M, S. Dresselhaus, G. D. \& A. Jorio. Group Theory: Application to the Physics of Condensed Matter (Springer-Verlag, Berlin, Heidelberg, 2008).

26. Bolte, J. \& Keppeler, S. A semiclassical approach to the dirac equation. Ann. Phys. 274, 125-162 (1999)

27. Leger, J. R. \& Swanson, G. J. Efficient array illuminator using binary-optics phase plates at fractional-Talbot planes. Opt. Lett. 15, 288-290 (1990).

28. Talbot, H. F. Facts relating to optical science. No. IV, Philos. Mag. 9 (1836).

29. Cheng, Q., Jiang, W. X. \& Cui, T. J. Spatial power combination for omnidirectional radiation via anisotropic metamaterials. Phys. Rev. Lett. 108, 213903 (2012).

30. Lu, M.-H. et al. Negative birefraction of acoustic waves in a sonic crystal. Nature Mater. 6, 744-748 (2007).

31. Christensen, J. \& Javier Garcia de Abajo, F. Anisotropic metamaterials for full control of acoustic waves. Phys. Rev. Lett. 108, 124301 (2012).

32. Zhang, X. Observing zitterbewegung for photons near the dirac point of a twodimensional photonic crystal. Phys. Rev. Lett. 100, 113903 (2008).

33. Zhang, X. \& Liu, Z. Extremal transmission and beating effect of acoustic waves in two-dimensional sonic crystals. Phys. Rev. Lett. 101, 264303 (2008).

34. Krutyanskiy, V. et al. Plasmonic enhancement of nonlinear magneto-optical response in nickel nanorod metamaterials. Phys. Rev. B 87, 035116 (2013).

35. Suchowski, H. et al. Phase mismatch-free nonlinear propagation in optical zeroindex materials. Science 342, 1223-1226 (2013).

\section{Acknowledgments}

The work was jointly supported by the National Basic Research Program of China (Grant No. 2012CB921503 and No. 2013CB632904) and the National Nature Science Foundation of China (Grant No. 1134006). We also acknowledge the support from Academic Program Development of Jiangsu Higher Education (PAPD) and China Postdoctoral Science Foundation (Grant No. 2012M511249 and No. 2013T60521). KAUST Baseline Research fund is also acknowledged.

\section{Author contributions}

Z.G.C. carried out the numerical simulations and theoretical analysis. X.N., X.C.S. and L.Y.Z. contributed in the discussion of theoretical analysis. M.H.L. and Y.F.C. conceived and supervised the study. Z.G.C., Y.W., C.H. and M.H.L. wrote the paper.

\section{Additional information}

Competing financial interests: The authors declare no competing financial interests.

How to cite this article: Chen, Z.-G. et al. Accidental degeneracy of double Dirac cones in a phononic crystal. Sci. Rep. 4, 4613; DOI:s10.1038/srep04613 (2014).

This work is licensed under a Creative Commons Attribution-NonCommercialNoDerivs 3.0 Unported License. The images in this article are included in the article's Creative Commons license, unless indicated otherwise in the image credit; if the image is not included under the Creative Commons license, users will need to obtain permission from the license holder in order to reproduce the image. To view a copy of this license, visit http://creativecommons.org/licenses/by-nc-nd/3.0/ 